\title{
LAS ELECCIONES AL PARLAMENTO VASCO DE 25 DE OCTUBRE DE 1998
}

\author{
CARLOS VIDAL PRADO
}

Profesor Asociado del Departamento de Derecho Constitucional. UNED 


\section{SUMARIO}

1. Una NUEVA Situación: elecCiones con tRegua. 2. La campaña electoral. a) Los mensajes de los partidos. b) Las encuestas. 3. LAS ELECCIONES. a) Resultados globales. b) Resultados por Territorios Históricos. 4. GeStIONAR LA PAZ: CONCLUSIONES. 


\section{LAS ELECCIONES AL PARLAMENTO VASCO DE 25 DE OCTUBRE DE 1998}

POR

CARLOS VIDAL PRADO

Profesor Asociado del Departamento de Derecho Constitucional. UNED

\section{UNA NUEVA SITUACIÓN: ELECCIONES CON TREGUA}

Las elecciones vascas de octubre de 1998 han tenido un significado especial, puesto que han sido las primeras en nuestra reciente historia democrática que han podido celebrarse en aquella Comunidad Autónoma sin la losa del terrorismo. El anuncio de abandono temporal de las armas por parte de ETA fue, sin duda, un elemento determinante y decisivo de cara al resultado de estas últimas elecciones. Los ciudadanos vascos podían ejercer, por primera vez, su derecho al voto, sin la tensión de saber que algunos podian emplear sus armas para condicionar el proceso democrático.

Ante la tregua anunciada por los terroristas un mes y medio antes de los comicios se produjeron las más diversas reacciones en la clase política vasca y española. Por un lado, todos se alegraban de que, por fin, se pudiese ver la luz al final del túnel. Por otro lado, la prudencia obligaba a no lanzar las campanas al vuelo. Los asesinos habían declarado otras treguas que, a la postre, se habían malogrado.

En esta ocasión, sin embargo, se daban algunos elementos que hacian pensar que la situación no era la misma. Por un lado, la repercusión internacional del anuncio de la tregua había sido impresionan- 
te. Con el reciente modelo del proceso de paz irlandés, en el exterior de nuestras fronteras se vincularon ambos problemas, de un modo incorrecto, puesto que no pueden simplificarse de esta manera situaciones tan complejas y diferentes como la vasca y la irlandesa. Pero el hecho es que la presión internacional hacia un proceso de paz era muy fuerte, y que ETA perderia todo tipo de apoyos y comprensiones externas (si es que alguna vez los había tenido) en caso de que volviese a las armas.

En segundo lugar, pronto se pudo detectar que detrás de los hechos conocidos se presentaban otros acontecimientos o compromisos no tan claros ante la opinión pública. La coincidencia en el tiempo de la tregua con la Declaración de Estella, firmada por los partidos nacionalistas (incluido HB) y por IU, no era casual. En dicha Declaración se planteaba la necesidad de reformar la Constitución y el Estatuto vasco, para reconocer el derecho a la autodeterminación de Euskadi. Parece que esta Declaración fue parte del pacto entre PNV, ETA y $\mathrm{HB}$, en el que, a cambio, ETA se comprometía a dejar temporalmente las armas. El problema era saber hasta dónde había llegado el PNV en dichas negociaciones, que había dirigido el portavoz Joseba Egibar. ¿A qué se había comprometido el PNV a cambio de la tregua? ¿Sólo a firmar la Declaración de Estella? Éste fue uno de los temas recurrentes durante la campaña.

Para poder saber si la tregua era real y sincera se requeria tiempo. Los partidos, llamados a consultas por el Presidente del Gobierno de la Nación, José María Aznar, estuvieron de acuerdo en posponer el debate político sobre la cuestión hasta después de las elecciones vascas. Pero el hecho cierto era que, al menos inicialmente, la tregua parecía beneficiar a $\mathrm{HB}$, lo cual indicaba que, desde el punto de vista estratégico, habían escogido el momento oportuno.

El brazo político de ETA había ido disminuyendo su apoyo social de manera alarmante para sus dirigentes. El cerco judicial se había ido estrechando, la Mesa Nacional se encontraba en la cárcel desde hacía meses, el diario Egin había sido clausurado... La imagen de HB se había radicalizado, y esto les había causado una gran pérdida de votos, reflejada en las encuestas. Se trataba, por tanto, de moderar la imagen. La tregua fue un elemento decisivo para ello. Además, ante el temor de una ilegalización de las siglas (tras el cierre del diario Egin se percibía ese riesgo), cambiaron el nombre de su coalición. Euskal Herritarrok (EH) era la heredera de HB, pero se presentaron como una coalición más abierta y moderada, con hipotéticas nuevas caras (aunque había, por supuesto, presos de ETA en sus listas, como siempre). El hecho es 
que parecia que esta imagen de moderación les habia salido rentable, al menos en las encuestas.

Otros beneficiados de este clima de paz, o de preparación para la paz, deberian ser los partidos nacionalistas, especialmente el PNV, que intentó capitalizar el proceso. Sin embargo, las encuestas no daban esos datos, como veremos en el próximo apartado. Los partidos no nacionalistas plantearon al PNV durante la campaña, en múltiples ocasiones, que explicase qué había concedido a HB y ETA en sus negociaciones, que dijese exactamente si el PNV perseguía o no la independencia. Con esta pregunta intentaban forzar al partido nacionalista a una radicalización del mensaje que llevase a sus electores más moderados a modificar el sentido de su voto, respaldando al PSOE o al PP. EI PNV no respondió a esas preguntas.

\section{LA CAMPAÑA ELECTORAL}

a) Los mensajes de los partidos

Como ya se ha dicho, los partidos no nacionalistas, fundamentalmente PP y PSOE, pidieron al PNV durante la campaña cuáles habían sido sus concesiones a HB y ETA en sus periódicas reuniones a lo largo de los últimos meses. En concreto, si su objetivo final era la independencia. EI PNV no contestó a estas preguntas. Sabia que un sector de sus votantes no vería con buenos ojos una radicalización del mensaje, y podía acabar votando al PP o al PSOE.

Desde el PSOE y PP se insistió en estas tesis. El Ministro del Interior, Jaime Mayor Oreja llegó a afirmar que el PNV había asumido los postulados de HB, lo cual fue respondido por Atutxa (Consejero del Interior del Gobierno Vasco, propuesto para futuro Presidente del Parlamento autónomo), en tono de reproche, no sólo negándolo, sino además manifestando que el Ministro "sabe que no es verdad". Sin embargo, en el interior del PNV no estaban claras estas posturas. Parece constatable que, desde hace años, existen dos corrientes, una moderada y otra más radical en dicho partido nacionalista. Atutxa se encuadraría en la primera de esas corrientes. Egibar - y quizá Arzallusen la más radical. Si se trataba de una estrategia en la cual a cada uno le correspondía ese rol, o si realmente sus posicionamientos públicos respondian a sus íntimas convicciones, no era fácil de saber. 
Por su parte, el candidato del PNV, Juan José lbarretxe, respondía más bien a la imagen moderada. No obstante, resultaba una incógnita cuál podía ser su rendimiento como cabeza de cartel electoral. Sus escasos conocimientos de euskera supusieron un hándicap al inicio de su carrera hacia la Presidencia, pero después de unos meses de clases intensivas de la lengua vasca, consiguió durante la campaña dirigir algunos discursos integros en euskera a sus simpatizantes. Ibarretxe si había demostrado ser un buen negociador con el Gobierno Aznar, ya desde el momento de la investidura del Presidente del Gobierno. Esto parecía augurar una buena relación futura entre los nacionalistas y el Partido Popular.

Los dos grandes partidos nacionales afrontaron la campaña con optimismo, por el resultado ascendente que les anunciaban las encuestas, pero con preocupación, ante la posibilidad de un frente nacionalista que formase un gobierno sin contar con PP y PSOE. Esta posibilidad fue sugerida en diversos artículos de opinión: quizá PNV y EA podrían formar gobierno sin mayoría absoluta, pero en connivencia con $\mathrm{EH} / \mathrm{HB}$.

Los populares llegaban a las elecciones después de haber perdido en el camino a un buen número de compañeros asesinados por ETA. Inicialmente, este hecho y la imagen de moderación del partido a nivel nacional podrían haberles dado más escaños en todas las provincias vascas, pero sin embargo su ascenso en las encuestas procedía exclusivamente de Álava, al recoger los votos $y$ escaños perdidos por Unidad Alavesa. Divisiones internas del partido en Guipúzcoa - que provocaron problemas con las listas electorales - y quizá la falta de tirón del candidato - que necesitó en demasía la presencia de Aznar, Mayor Oreja y otros líderes nacionales - podían provocar este moderado ascenso.

Los socialistas se encontraban en una fase de prudente recuperación, tras el fracaso electoral de 1994. El candidato, Nicolás Redondo, recurrió también a la ayuda y apoyo de los líderes nacionales durante la campaña. Además, el PSE-PSOE incrementó sus ataques al PNV, intentando quizá restar protagonismo como defensor de los intereses españoles al PP, como partido en el Gobierno. La agresividad entre PSOE y PNV fue bastante alta, hasta que en la última semana de campaña algunos líderes socialistas la moderaron, reconociendo incluso, como hizo Borrell, la necesidad de contar con el PNV para formar un gobierno en Euskadi.

1 Isabel San Sebastián, $A B C, 19-X-98$, p. 26. 
Durante los dias previos al domingo en que se celebraron las elecciones, dos elementos incidieron en el desarrollo de la campaña. Uno de ellos, externo, provocó que se hablase mucho menos de las elecciones vascas en los medios de comunicación, especialmente los de ámbito nacional: se trataba de la petición de extradición a España del general Pinochet (que se encontraba en Londres), por parte del juez Garzón. El otro elemento también tuvo su origen en Londres. La televisión británica anunciaba para el sábado 24 de octubre, día de reflexión, un programa sobre el problema vasco en el que, al parecer, iban a intervenir dos encapuchados de ETA, con un mensaje al electorado vasco.

El contenido de dicho mensaje fue motivo de controversias entre los líderes políticos. Se esperaba una confirmación de la tregua por parte de ETA. Además, esto se unía a los rumores que apuntaban a la celebración en Bélgica de una Asamblea de ETA. En fin, la banda terrorista consiguió capitalizar la atención en la recta final de la campaña, aunque al final la intervención de ETA en la televisión británica no aportó ninguna novedad.

\section{b) Las encuestas}

Pocas elecciones como éstas habian despertado tanto interés en los últimos años. Ello se vio reflejado en la gran cantidad de encuestas que, encargadas desde diversos sectores, se fueron haciendo públicas durante la campaña. Incluso la cadena de televisión Tele 5 estableció un barómetro electoral, que, día a día, aportaba los datos de evolución de la opinión pública, traducidos en número de escaños para los partidos. De esta encuesta se encargaba el Instituto Ábaco.

Otros medios de comunicación, tanto nacionales como vascos, publicaron una o varias encuestas durante la campaña. Como siempre, el domingo anterior a las elecciones fue el que más consultas demoscópicas aportó. Las grandes empresas especializadas en este tipo de estudios, tanto nacionales como vascas, se vieron inmersas en una frenética actividad durante estos días. También el Centro de Investigaciones Sociológicas y el Gobierno Vasco hicieron públicas sus propias encuestas.

Desde el momento en que se anunció la tregua, y siempre según lo indicado en las encuestas, se pudieron constatar dos tendencias diferentes en la evolución de la opinión pública vasca. Los primeros días fueron de claro ascenso nacionalista. Tanto el PNV como EH/HB incrementaron de modo considerable sus expectativas de voto. Especial- 
mente llamativo fue el caso de $\mathrm{EH} / \mathrm{HB}$, que no sólo detuvo el constante descenso en apoyos populares, sino que los incrementó considerablemente, hasta llegar a situarse en las encuestas como segunda fuerza política de Euskadi. Junto a este ascenso nacionalista, se daba un descenso o estancamiento de los partidos nacionales (PP, PSOE e IU) y un claro descenso de Unidad Alavesa.

Sin embargo, esta tendencia inicial se invirtió ya durante la campaña. EI PNV fue bajando paulatinamente, de modo que las encuestas le adjudicaban un número de escaños similar o inferior al que ya tenían (21 ó 22). EH/HB también disminuyó su intención de voto, hasta alcanzar niveles similares a los de la consulta de 1994, quedándose en 11 ó 12 escaños. De modo simétrico, los partidos nacionales incrementaban sus expectativas, pugnando por la segunda plaza el PP y el PSOE, que podrían alcanzar entre 13 y 15 escaños.

Esta última tendencia se confirmó en la mayoría de las encuestas publicadas una semana antes de las elecciones, el domingo 18 de octubre. Así, las de Gallup en $A B C$, Demoscopia en EI País y la de EI Correo Español-El Pueblo Vasco. Sin embargo, la encuesta de Sigma-Dos para El Mundo continuaba otorgando la segunda posición a $E H / H B$, por delante de PP y PSOE².

Un dato común a todas las encuestas era el estancamiento de IU, que mantuvo posturas un tanto ambiguas con respecto a las posturas independentistas (como se ha dicho, fue el único partido no nacionalista que firmó el pacto de Estella-Lizarra), quizá para arañar voto abertzale de izquierdas, y sin embargo no lograba su objetivo. Otro dato similar en los estudios de opinión era el mantenimiento de la tendencia a la baja de EA, perdiendo uno o dos escaños con respecto a 1994. Unidad Alavesa bajaba también estrepitosamente, pasando de 5 escaños con un 19\% de voto en Álava, a 2 escaños con sólo un 9\%. De este descenso en votos de los foralistas alaveses se beneficiaba el PP, que los recogía casi en su totalidad.

La valoración personal del presidente del PNV una semana antes de las elecciones era bastante clara: "La abstención va a bajar; nosotros tenemos 22, incrementando bastante los votos; subirá fuertemente HB por efecto de la tregua; el PP subirá lo que rescate de UA, y no

2 La posibilidad de que HB fuese la segunda fuerza politica fue objeto de comentarios durante la campaña. Asi, por ejemplo, Arzallus dijo el 18 de octubre en Vitoria: «Sería tremendo para los que enviaron a la cárcel a la Mesa de HB que este partido quedase como segunda fuerza polítican ( $A B C, 19-X-98, p .23)$. 
mucho más»3. A tenor de lo que decían las encuestas, parecía no apartarse mucho de la realidad la frase de Arzallus. Sin embargo, un dato reflejado en la ficha técnica de las consultas dejaba la puertas abiertas a posibles modificaciones de los resultados. No sólo había muchos indecisos, sino que también muchos ciudadanos vascos no querían desvelar su opinión al ser preguntados. Significativa actitud, que reflejaba sin duda la presión con que se vivía esta campaña, a pesar de que los terroristas no estuviesen actuando ${ }^{4}$.

\section{LAS ELECCIONES}

\section{a) Resultados globales}

El número de electores convocados a las urnas era de 1.821.459, de los cuales ejercieron su derecho al voto un $70,7 \%$ del total. La cifra es la más alta de todas las elecciones autonómicas celebradas hasta la fecha en Euskadi. Ya durante el día, los datos de participación que el Gobierno Vasco iba ofreciendo eran muy significativos. La llamada a las urnas de los partidos (especialmente del PP y PSOE) fue eficaz. Se trataba de movilizar a muchos electores que, acostumbrados a votar en las generales, no lo hacían en las autonómicas. Teniendo en cuenta que el PP y PSOE mejoran resultados siempre en las generales, una mayor participación parecía augurar un mejor resultado para los dos grandes partidos nacionales ${ }^{5}$. Sin embargo, alguna de las encuestas celebradas a la salida de los colegios electorales sembró el desconcierto en los primeros momentos, pues atribuyó a HB/EH la condición de segunda fuerza política, con un número de escaños que podía oscilar entre 16 y $18^{6}$.

En la siguiente tabla podemos observar los diferentes porcentajes de participación que ha habido hasta ahora en el País Vasco?.

$3 \quad$ Cfr. $A B C, 19-\mathrm{X}-98$, p. 23.

4 A la pregunta "¿Qué partido cree que va a ganar estas elecciones?», de la encuesta realizada por Gallup, un $16,5 \%$ No sabe/No contesta (Cfr. $A B C, 18-X-98$, p. 35.). Más significativa todavía es la ausencia de respuesta a la pregunta "¿Qué partido le gustaría que ganara?", de la encuesta de Demoscopia para El País: el No sabe/No contesta alcanza el $30 \%$, el mismo porcentaje de los que muestran sus preferencias por una victoria del PNV (Cfr. El Pais, 18-X-98, p. 18).

5 Algunos los llamaron "autonomistas» o «constitucionalistas», por su defensa del marco jurídico representado por el Estatuto de Autonomía y la Constitución, frente a las posiciones críticas de PNV, EA y EH/HB.

6 Los sondeos encargados por diversos medios de comunicación a diversas empresas demoscópicas sorprendieron por su gran diversidad. Fue especial- 
EVOLUCIÓN DE LA PARTICIPACIÓN

\begin{tabular}{|c|c|c|}
\hline & Autonómicas & Generales \\
\hline $1977 \ldots \ldots \ldots \ldots \ldots \ldots \ldots \ldots$ & & 76,9 \\
\hline 1979 & & 65,9 \\
\hline $1980 \ldots \ldots \ldots \ldots \ldots \ldots \ldots$ & 59,76 & \\
\hline $1982 \ldots \ldots \ldots \ldots$ & & 79,37 \\
\hline 1984 & 68,49 & \\
\hline 1986 & 69,62 & 67,58 \\
\hline 1989 & & 66,89 \\
\hline 1990 & 60,99 & \\
\hline 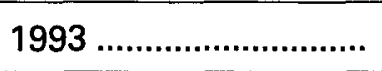 & & 69,63 \\
\hline 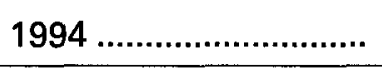 & 59,69 & \\
\hline 1996 & & 71,53 \\
\hline 1998 & 70,7 & \\
\hline
\end{tabular}

Como puede apreciarse, la participación se situó en niveles semejantes a los de unas elecciones generales, lo cual motivó una positiva reacción de todos los partidos en sus primeras declaraciones.

En la siguiente tabla se recogen los resultados de los principales partidos políticos. Al señalar los datos de 1994 se recoge entre paréntesis la diferencia de los datos de 1998 con respecto a 1994.

mente llamativo el de ECO-Consulting para TVE, que otorgaba a HB la segunda posición, con un número de escaños que podía oscilar entre 16 y 18 . Las criticas de los analistas políticos arreciaron, e incluso llegaron a acusar a los encuestadores de que, en ocasiones, se realiza una asignación preconcebida del voto, cuando se encuentran bolsas de encuestados que no quieren revelar su opinión. Sin embargo, se ha de tener en cuenta también el fenómeno de ocultación de voto, que perjudica siempre (en las encuestas) a los partidos no nacionalistas, especialmente PP y PSOE. Un votante de HB no tiene ningún reparo en manifestar la dirección de su voto, pero el del PP no lo hace con tanta facilidad. Como hemos comentado, este fenómeno ya se producía en las encuestas realizadas durante la campaña.

7 Salvo que indiquemos lo contrario, todos los datos que figuran en este trabajo se han obtenido del Gobierno Vasco. 


\begin{tabular}{|c|c|c|c|c|c|c|}
\hline Candidatura & Votos 1998 & $\% 1998$ & Esc. 1998 & Votos 1994 (Dif.) & \% 1994 (Dif.) & Esc. 94 (Dif.) \\
\hline EAJ-PNV.......... & 347.958 & 27,97 & 21 & $304.346(+43.612)$ & $29,84 \quad(-1,87)$ & $22(-1)$ \\
\hline PSE-EE ........... & 218.607 & 17,57 & 14 & $174.682(+43.925)$ & $17,13(+0,44)$ & $12(+2)$ \\
\hline 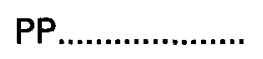 & 250.580 & 20,14 & 16 & $146.960(+103.620)$ & $14,41 \quad(+5,73)$ & $11(+5)$ \\
\hline EA $\ldots \ldots \ldots \ldots \ldots \ldots$ & 108.300 & 8,70 & 6 & $105.136(+3.164)$ & $10,31 \quad(-1,61)$ & $8(-2)$ \\
\hline HB/EH ............. & 223.264 & 17,94 & 14 & $166.147(+57.117)$ & $16,29(+1,67)$ & $11(+3)$ \\
\hline IU/EB................ & 70.835 & 5,69 & 2 & $93.291 \quad(-22456)$ & $9,15(-3,46)$ & $6(-4)$ \\
\hline 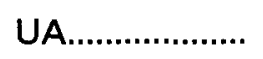 & 15.722 & 1,26 & 2 & $27.797(-12.075)$ & $2,73(-1,47)$ & $5(-3)$ \\
\hline
\end{tabular}


A la vista de estos datos, podemos obtener ya algunas conclusiones, aunque convendrá desglosar los votos por provincias para poder establecer una valoración más precisa. No obstante, es clara la victoria del PNV, con la pérdida de un escaño. Aunque incrementa el número de votos, el porcentaje obtenido sobre el total es menor que en 1994 , y pierde un $1,87 \%$.

Por otro lado, el Partido Popular incrementa sus votos de modo espectacular, y no sólo a costa de Unidad Alavesa, sino que también sube en los otros dos Territorios Históricos, hasta situarse como segunda fuerza política del País Vasco.

El otro gran vencedor de las elecciones es EH/HB. Aunque pareciese éste un dato inquietante, pienso que no es así. Es evidente que la ruptura de la tendencia a la baja de HB se produce cuando ETA anuncia el abandono de las armas. Si el sector abertzale radical recupera muchos de los votos perdidos, es sin duda porque electores que les habian abandonado vuelven a votarles, pero con una exigencia: dejar los métodos violentos. $\mathrm{EH} / \mathrm{HB}$ recibe de esta manera un mandato muy claro en las urnas, que probablemente será decisivo para convertir una tregua que podia parecer provisional en definitiva.

Como predecian las encuestas, UA sufre un importante retroceso. Pero también lo experimenta IU, lo cual no estaba previsto, ni siquiera en los sondeos que se hicieron a la salida de los colegios electorales 8 . Por su parte, el PSOE mejora posiciones, aunque sin llegar a niveles que habían alcanzado en los años 80 , y pasando de ser segunda a cuarta fuerza política de Euskadi.

Pasemos sin embargo a ver los resultados en cada Territorio Histórico, porque de esta manera se puede analizar mejor el trasvase de votos entre unas fuerzas y otras.

\section{b) Resultados por Territorios Históricos}

(Vénase tablas en las páginas 413,414 y 415.$)$

s En este caso también fallaron los sondeos a pie de urna, pues ninguno de ellos vaticinó el descenso de IU, sino que le asignaban un número de escaños similar (con la pérdida sólo de un diputado) al de las últimas elecciones de 1994. 
ÁlAVA

\begin{tabular}{|c|c|c|c|c|c|c|c|}
\hline & Votos 1998 & \% 1998 & $\% 1994$ & $\begin{array}{c}\text { Variac. } \\
\%\end{array}$ & $\begin{array}{l}\text { Esc. } \\
1998\end{array}$ & $\begin{array}{l}\text { Esc. } \\
1994\end{array}$ & $\begin{array}{c}\text { Variac. } \\
\text { esc. }\end{array}$ \\
\hline EAJ-PNV ........... & 36.830 & 21,95 & 22,11 & $-0,16$ & 6 & 6 & $=$ \\
\hline PSE-EE ........... & 28.574 & 17,03 & 15,84 & $+1,19$ & 5 & 4 & +1 \\
\hline PP ........................ & 45.273 & 26,98 & 16,18 & $+10,80$ & 7 & 4 & +3 \\
\hline EA ........................ & 10.700 & 6,38 & 7,36 & $-0,98$ & 1 & 2 & -1 \\
\hline HB/EH ............... & 20.536 & 12,24 & 10,25 & $+1,99$ & 3 & 2 & +1 \\
\hline IU/EB ................. & 9.625 & 5,74 & 9,23 & $-3,49$ & 1 & 2 & -1 \\
\hline UA ...................... & 14.278 & 8,51 & 18,83 & $-10,32$ & 2 & 5 & -3 \\
\hline
\end{tabular}


VIZCAYA

\begin{tabular}{|c|c|c|c|c|c|c|c|}
\hline & Votos 1998 & \% 1998 & $\% 1994$ & $\begin{array}{c}\text { Variac. } \\
\%\end{array}$ & $\begin{array}{l}\text { Esc. } \\
1998\end{array}$ & $\begin{array}{l}\text { Esc. } \\
1994\end{array}$ & $\begin{array}{c}\text { Variac. } \\
\text { esc. }\end{array}$ \\
\hline EAJ-PNV ............... & 222.902 & 32,99 & 35,66 & $-2,67$ & 9 & 10 & -1 \\
\hline PSE-EE ................. & 125.022 & 18,50 & 17,66 & $+0,84$ & 5 & 4 & +1 \\
\hline PP & 138.642 & 20,52 & 15,27 & $+5,25$ & 5 & 4 & +1 \\
\hline 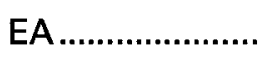 & 41.011 & 6,07 & 7,20 & $-1,13$ & 1 & 2 & -1 \\
\hline 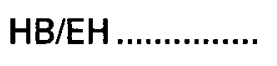 & 99.936 & 14,79 & 13,60 & $+1,19$ & 4 & 3 & +1 \\
\hline IU/EB & 42.576 & 6,30 & 10,21 & $-3,91$ & 1 & 2 & -1 \\
\hline
\end{tabular}


GUIPÚZCOA

\begin{tabular}{|c|c|c|c|c|c|c|c|}
\hline & Votos 1998 & $\% 1998$ & $\% 1994$ & $\begin{array}{c}\text { Variac. } \\
\%\end{array}$ & $\begin{array}{l}\text { Esc. } \\
1998\end{array}$ & $\begin{array}{l}\text { Esc. } \\
1994\end{array}$ & $\begin{array}{c}\text { Variac. } \\
\text { esc. }\end{array}$ \\
\hline EAJ-PNV ........... & 88.226 & 22,02 & 22,79 & $-0,77$ & 6 & 6 & $=$ \\
\hline PSE-EE ............. & 65.011 & 16,22 & 16,74 & $-0,52$ & 4 & 4 & $=$ \\
\hline PP $\ldots \ldots \ldots \ldots \ldots \ldots \ldots$ & 66.665 & 16,64 & 12,14 & $+4,50$ & 4 & 3 & +1 \\
\hline EA ..................... & 56.589 & 14,12 & 17,08 & $-2,96$ & 4 & 4 & $=$ \\
\hline HB/EH ............... & 102.792 & 25,65 & 23,63 & $+2,02$ & 7 & 6 & +1 \\
\hline IU/EB ................. & 18.634 & 4,65 & 7,23 & $-2,58$ & 0 & 2 & -2 \\
\hline
\end{tabular}


En Álava se produce el anunciado trasvase de votos entre PP y UA. Tanto en número de escaños como en porcentajes es prácticamente igual el incremento del PP $(+10,80 \%)$ y el descenso de UA $(-10,32 \%)$. Los foralistas de Álava pierden tres escaños, que son exactamente los que gana el PP. Sin embargo, en número de votos no ocurre lo mismo. UA pierde alrededor de once mil, mientras que el PP gana veinticuatro mil. Esto quiere decir que los populares recogen también nuevos apoyos, bien de electores que participan por primera vez en unas autonómicas, bien procedentes de otros partidos. Todo ello trae consigo que el PP se convierte en la primera fuerza política de este Territorio Histórico.

Las otras modificaciones que se dan en esta provincia parecen también explicables. IU pierde un escaño, que probablemente se corresponde con el que gana el PSOE. EA pierde otro escaño, que seguramente será el que ha ido a parar a manos de $\mathrm{HB} / \mathrm{EH}$.

Por lo demás, destaca el triunfo del PP en Vitoria, con un $29 \%$ de los votos, frente al $18,6 \%$ del PSOE y el $17,8 \%$ del PNV. Unidad Alavesa concentra casi todos sus votos en la capital. De los 14.278 que logra en la provincia, 12.553 los consigue en Vitoria.

Por su parte, Vizcaya continúa siendo feudo del PNV, aunque es aquí donde pierden un escaño, y tanto el PP como el PSOE avanzan en número de votos. Los socialistas, sin embargo, no recuperan cotas que habían logrado en la década de los 80 , a pesar de que su candidato a lehendakari, Nicolás Redondo, fuese vizcaíno.

Se produce en Vizcaya una ligera modificación en la distribución de escaños entre partidos nacionalistas y nacionales. Los primeros pierden un escaño en beneficio de los segundos. Probablemente se pueda tratar del escaño del PNV, que quizá haya pasado a manos del PP. El diputado que pierde Izquierda Unida es probablemente el que gana el PSOE, $y$ el que pierde EA lo recoge HB/EH.

En la ciudad de Bilbao gana de nuevo el PNV, con el $30,8 \%$ de los votos. Sin embargo, el PP. queda muy cerca, con un $26,8 \%$, y a sólo ocho mil quinientos votos.

Guipúzcoa sigue siendo dominio de $\mathrm{HB} / \mathrm{EH}$, que incrementa su ventaja sobre el PNV. Los travases de escaños están provocados, en esta ocasión, no sólo por la diferente distribución de voto, sino por otro elemento añadido. IU no alcanza el $5 \%$ mínimo para acceder al reparto de escaños. Esto quiere decir que, aunque proporcionalmente le corresponderia al menos un escaño, no lo recibe, al quedarse por debajo 
de la barrera electoral. Así pues, pierde los dos que tenía, y esto contribuye a engrosar el número de escaños a repartir entre los demás partidos. Uno lo recibe HB/EH y el otro el Partido Popular. En este caso, no se pueden establecer correspondencias ideológicas entre los escaños, por las razones anteriormente apuntadas. Si Izquierda Unida hubiese superado el $5 \%$, habría logrado un escaño, en detrimento del PP. Lo que sí puede aventurarse es que el segundo escaño perdido por lzquierda Unida va a parar a manos de HB/EH.

Destaca en esta provincia el contundente triunfo del Partido Popular en San Sebastián. Consigue más de veintisiete mil votos, con un $25,7 \%$. El siguiente partido es $\mathrm{HB} / \mathrm{EH}$, con veinte mil votos y un $19,3 \%$. Después, con dieciocho mil (17\%) se sitúan PNV y PSOE. Me parece interesante contrastar este dato con el de algunas poblaciones de Guipúzcoa en las cuales el dominio de HB es aplastante, y que nos llevan a cuestionar la libertad de los que ejercen allí su derecho al voto. Así por ejemplo, en Ataun, los resultados son los siguientes: HB, 575; PNV, 322; EA, 196; PSE, 10; PP, 10; IU, 10. Pues bien, los tres últimos partidos, a pesar de conseguir esas ridículas cantidades de votos, jmejoran sus resultados! En 1994 sólo llegaro a conseguir 6, 3 y 4 votos, respectivamente. Sin irnos a un lugar tan recóndito, en Guetaria encontramos unos resultados bastante similares: HB, 544; PNV, 699; EA, 299; PP, 47; PSOE, 24; IU, 20. Es decir, que frente a 1.542 votos nacionalistas, nos encontramos 91 votos no nacionalistas. Se comprende quizá mejor, a la vista de estos resultados, la dificultad que supone votar a un partido no nacionalista en zonas donde existe una fuerte tradición nacionalista, en las que, en ocasiones, la presión en este sentido puede ser considerable.

\section{GESTIONAR LA PAZ: CONCLUSIONES}

Se dan en estas elecciones vascas una serie de elementos que las significan como decisivas de cara a la resolución del problema quizá más grave de la democracia española. Como decía al inicio de este comentario, la tregua provisional declarada antes de las elecciones por ETA podia convertirse o no en definitiva en función de los resultados electorales, en primer lugar, y de la administración de esos resultados por parte de los partidos, en segundo término.

Un primer dato relevante, como ya se ha comentado, es el récord de participación. Es un signo claro de que el pueblo vasco ha asumido 
el reto, y le está diciendo a sus políticos que quieren la paz, y que delegan en ellos la manera de conducir la situación para que ésta se convierta en definitiva.

Esta elevada participación no se traduce, sin embargo, en un cambio sustancial en el mapa político vasco. Los escaños ocupados por fuerzas nacionalistas (41) y no nacionalistas (34) se mantienen exactamente igual que en las elecciones de 1994. No obstante, en número de votos totales sí se observa una tendencia al alza mayor entre los partidos no nacionalistas que en los otros. En la tabla siguiente se puede comprobar este dato.

\begin{tabular}{lccccc}
\hline $\begin{array}{l}\text { Elecciones } \\
\text { Autonómicas }\end{array}$ & 1980 & 1986 & 1990 & 1994 & 1998 \\
\hline Nacionalistas ............... & 590.691 & 776.706 & 670.919 & 574.122 & 678.968 \\
\hline No nacionalistas.......... & 288.912 & 355.034 & 321.926 & 441.319 & 555.327 \\
\hline
\end{tabular}

En definitiva, se puede afirmar que a pesar del cierto carácter plebiscitario que algunos partidos quisieron otorgar a estas elecciones, el electorado vasco no refrenda ese carácter, sino que su mandato a los partidos es de pacto y negociación. La formación de gobierno no será tarea fácil, pero los partidos deberán secundar la intención de los electores.

Lo que sí cambia es la distribución dentro de los dos grandes grupos de partidos. En el ámbito nacionalista, HB/EH capitaliza la tregua y el Pacto de Estella, ganando tres escaños, los mismos que pierden PNV y EA. Sin embargo, como hemos visto, no existe una correlación exacta entre los escaños que pierden unos y gana otro. Para $\mathrm{HB} / \mathrm{EH}$ este incremento de votos supone un mensaje de su electorado, en el sentido de abandonar definitivamente la violencia y utilizar los cauces políticos democráticos para conseguir sus fines políticos. No debería haber vuelta atrás en el camino que los radicales han tomado, puesto que sería una traición al electorado.

En el ámbito de los partidos no nacionalistas, el que se beneficia de la nueva situación es el PP, que gana cinco escaños. Es significativo el hecho de que, incluso, rompe el techo de las elecciones generales de 
1996. En dicha convocatoria habían conseguido 231.284 votos, y ahora obtienen casi veinte mil votos más. Teniendo en cuenta que la tendencia en las autonómicas es siempre más favorable a los partidos nacionalistas, este resultado tiene especial relevancia. Quizá sea esto un reconocimiento a la labor de sus concejales y cargos electos, que hasta el momento del alto el fuego habían estado en el punto de mira, y un buen grupo de ellos había sido asesinado. Respaldan también los electores vascos la gestión de la tregua por parte del Gobierno del PP en Madrid.

PSOE recupera dos parlamentarios, aunque quizá sus dirigentes esperaban un mayor incremento del voto. De hecho, a pesar de subir, pasan de ser la segunda a la cuarta fuerza de Euskadi. Izquierda Unida se hunde, lo cual podría interpretarse como un castigo de los electores a un partido no nacionalista que, sin embargo, en los últimos meses había mantenido posturas ambiguas, acercándose a posiciones más proclives a la independencia y al derecho a la autodeterminación. Probablemente, los electores, con este castigo, le estaban diciendo a Izquierda Unida que ése no era su sitio y que debía volver a situarse en sus posiciones tradicionales.

Eusko Alkartasuna y Unidad Alavesa se ven perjudicados por la polarización del voto en las cuatro grandes opciones políticas, al igual que IU. Para los foralistas alaveses el futuro se presenta complicado, y sobre todo las elecciones municipales del segundo trimestre de 1999. En cuanto a EA, se ve probablemente perjudicada por el incremento de votos de $\mathrm{HB} / \mathrm{EH}, \mathrm{y}$ va perdiendo fuelle. No parece sino que este partido depende exclusivamente de su líder, Carlos Garaikoetxea. El momento de su retirada personal pondrá fin también a la trayectoria política de EA.

Al nuevo Gobierno le corresponde el difícil reto de dar los pasos oportunos para que la paz sea definitiva. 